\title{
Melanoma Maligno Cutâneo: Estudo Retrospetivo de Sete Anos (2006-2012)
}

\author{
Cutaneous Malignant Melanoma: a Retrospective Study of Seven Years \\ (2006-2012)
}

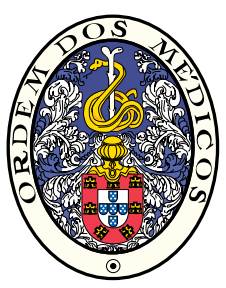

\author{
Jorge MOREIRA ${ }^{1,2}$, Elisabete MOREIRA², Filomena AZEVEDO², Alberto MOTA ${ }^{2,3}$ \\ Acta Med Port 2014 Jul-Aug;27(4):480-488
}

\section{RESUMO}

Introdução: O melanoma maligno é a neoplasia cutânea mais agressiva, e a sua incidência tem vindo a aumentar nas últimas décadas. A possibilidade de cura depende de um diagnóstico atempado, sendo fundamental o conhecimento da sua epidemiologia para a implementação de programas de prevenção primária e deteção precoce do melanoma.

Material e Métodos: Foi efetuada revisão dos processos clínicos dos doentes com melanoma maligno cutâneo primário, diagnosticados entre janeiro de 2006 e dezembro de 2012, no Centro Hospitalar de São João, Porto.

Resultados: Analisaram-se os 148 casos de melanoma diagnosticados neste período, tendo-se observado um predomínio do sexo feminino (razão F:M - 1,6:1). A média etária na altura do diagnóstico foi de 61 anos. As localizações mais frequentemente envolvidas foram os membros inferiores e o tronco. No sexo masculino o dorso foi o local mais afetado, enquanto no sexo feminino as lesões ocorreram, preferencialmente, nas pernas. O melanoma de extensão superficial foi o subtipo predominante em quase todas as faixas etárias. Verificou-se um predomínio dos melanomas finos e o índice mitótico foi intermédio (1-6 mitoses/ $\mathrm{mm}^{2}$ ) na maioria dos doentes. A ulceração esteve presente em $22,3 \%$ dos casos e predominou nos melanomas espessos e no subtipo nodular. A maioria dos doentes encontrava-se no estádio IA. A progressão para doença metastática ocorreu em 20 doentes.

Discussão: O perfil do doente com melanoma cutâneo, no Centro Hospitalar de São João, apresenta características relativamente semelhantes às descritas na literatura.

Conclusão: O predomínio dos melanomas finos, considerados de melhor prognóstico, é provavelmente, o resultado de um diagnóstico cada vez mais precoce.

Palavras-chave: Melanoma; Neoplasias da Pele; Estudos Retrospectivos; Portugal.

\section{ABSTRACT}

Introduction: Malignant melanoma is the most aggressive skin cancer and its incidence has been increasing in recent decades. The cure depends on early diagnosis and understanding melanoma epidemiology is of great importance for the implementation of programs of primary prevention and early detection.

Material and Methods: We reviewed the clinical cases of primary cutaneous malignant melanoma diagnosed between January 2006 and December 2012 at Centro Hospitalar de São João, in Porto.

Results: An overall of 148 cases of melanoma were analyzed and a female predominance was observed (ratio F:M - 1.6:1). The average age at diagnosis was 61 years. The locations most frequently affected were the lower limbs and trunk. In males the back was the most frequently involved, while in females the lesions occurred preferentially in the legs. Superficial spreading melanoma prevailed in almost all age groups. There was a predominance of thin melanomas and the mitotic index was intermediate (1-6 mitoses/ mm²) in most patients. The ulceration was present in $22.3 \%$ of cases and prevailed in thick melanomas as well in nodular subtype. Most patients present at stage IA. There was progression to metastatic disease in 20 patients.

Discussion: The profile of patients with cutaneous melanoma in Centro Hospitalar de São João has characteristics relatively similar to those described in the literature.

Conclusion: The prevalence of thin melanomas, generally with a better outcome, is probably the result of an earlier detection.

Keywords: Skin Neoplasms; Retrospective Studies; Portugal.

\section{INTRODUÇÃo}

O melanoma é uma neoplasia maligna com origem nos melanócitos, e ocorre preferencialmente na pele (> 90\% dos casos), embora, se possa observar igualmente nas mucosas, globos oculares ou leptomeninges. ${ }^{1}$

Apesar de representar apenas $4 \%$ dos cancros cutâneos é, no entanto, o mais agressivo, pelo seu elevado potencial de metastização, sendo responsável por $75 \%$ das mortes atribuídas a todas as neoplasias cutâneas. ${ }^{2}$

Numa altura em que a incidência de muitos tumores tem diminuído, o melanoma afigura-se continuar a aumentar em vários países, estimando-se que na população cau- casiana, este aumento seja de 3 a $7 \%$ ao ano. ${ }^{3} \mathrm{O}$ melanoma maligno, tornou-se assim, um importante problema de saúde pública.

São vários os fatores de risco ambientais e constitucionais associados ao melanoma e incluem, a saber, a exposição à radiação UV, os fototipos 1 e 2 de Fitzpatrick, os nevos melanocíticos (congénitos de grandes dimensões, múltiplos nevos melanocíticos adquiridos típicos e/ou nevos atípicos), história familiar ou pessoal de melanoma, genodermatoses, como o xeroderma pigmentosum, imunossupressão e história pessoal de cancro cutâneo não

\footnotetext{
1. Aluno. Mestrado Integrado em Medicina. Faculdade de Medicina. Universidade do Porto. Porto. Portugal.

2. Serviço de Dermatologia e Venereologia. Centro Hospitalar de São João, EPE. Porto. Portugal.

3. Faculdade de Medicina. Universidade do Porto. Porto. Portugal.

Recebido: 26 de Janeiro de 2014 - Aceite: 19 de Maio de 2014 | Copyright @ Ordem dos Médicos 2014
} 
melanoma. ${ }^{1,4}$

Nos fatores que influenciam o prognóstico estão incluídas características histopatológicas, das quais se destacam o índice de Breslow, a presença de ulceração e o índice mitótico. A idade, o sexo, e a invasão linfovascular são outros fatores reconhecidos. A localização anatómica e o seu impacto no prognóstico tem sido controverso, no entanto, alguns estudos sugerem que os melanomas do tronco, cabeça e pescoço têm pior prognóstico, comparativamente a outras localizações. ${ }^{1,5}$

A deteção precoce e o tratamento cirúrgico atempado impedem, na maioria dos casos, a progressão da doença, obtendo-se uma sobrevida superior a $90 \%$ aos cinco anos. No entanto, após o desenvolvimento de metástases, a sobrevida média é de apenas 6 a 9 meses. $^{6}$

O diagnóstico e tratamento precoces são, portanto, as melhores formas de abordar o doente com melanoma. O conhecimento das características epidemiológicas, clínicas e histopatológicas do melanoma maligno pode contribuir para aperfeiçoar a sua abordagem favorecendo, em particular, o seu diagnóstico precoce e, consequente, diminuição da mortalidade.

\section{MATERIAL E MÉTODOS}

Efetuou-se revisão dos processos clínicos dos doentes com melanoma maligno cutâneo primário, diagnosticados entre janeiro de 2006 e dezembro de 2012, no Centro Hospitalar de São João, EPE, Porto (CHSJ). A identificação dos casos foi fornecida pelo registo oncológico do CHSJ.

Foram analisadas as seguintes variáveis clínicas: sexo, idade no momento do diagnóstico, localização, sintomas e/ ou alterações da lesão primária, tempo de evolução da lesão, presença de nevos melanocíticos atípicos (nenhum; $\leq 10$ e > 10), imunossupressão, história familiar ou pessoal de melanoma ou de cancro cutâneo não melanoma, orientação terapêutica e evolução clínica. Foram, também, avaliadas as características histológicas: subtipo, índice de
Breslow, nível de Clark, ulceração, índice mitótico, invasão linfovascular ou neural, regressão tumoral, satelitose e presença de infiltrado linfocitário.

O estadiamento do melanoma foi baseado nas guidelines do American Joint Committee on Cancer (AJCC), $7^{\mathrm{a}}$ edição, de 2010.

Foi efetuada análise estatística dos dados utilizando o programa SPSS versão 21. As variáveis categóricas foram descritas através de frequências absolutas $(n)$ e relativas (\%). As variáveis contínuas foram descritas como média \pm desvio padrão. Foi usado o teste de independência do Qui-quadrado para analisar a associação entre variáveis categóricas. Quando mais de $20 \%$ da frequência esperada de células da tabela de contingência relativa à análise de associação de duas categorias foi inferior a 5, utilizou-se o teste exato de Fisher. Na ausência de cumprimento do pressuposto de homogeneidade das variâncias foi usado o teste de Welch. Em todos os testes de hipóteses foi considerado um nível de significância de 0,05. Foi efetuada a análise multivariada para avaliar a independência da variável ulceração como fator de risco de metastização. O risco relativo dos fatores preditivos foi acompanhado de intervalos de confiança de $95 \%$.

O presente estudo foi aprovado pela Comissão de Ética do CHSJ.

\section{RESULTADOS}

Entre 2006 e 2012 foram diagnosticados 148 casos de melanoma cutâneo primário, correspondentes a 91 mulheres $(61,5 \%)$ e 57 homens (38,5\%), todos caucasianos (Fig. 1).

A média etária no momento do diagnóstico foi de $61 \pm$ 17 anos (15-98 anos), com predomínio das faixas etárias dos 71 - 80 anos (23,6\%) e dos 51- 60 anos (18,9\%). A média de idades no sexo feminino foi ligeiramente superior à do sexo masculino, respetivamente, $62 \pm 18$ e $59 \pm 16$ anos (Tabela 1).

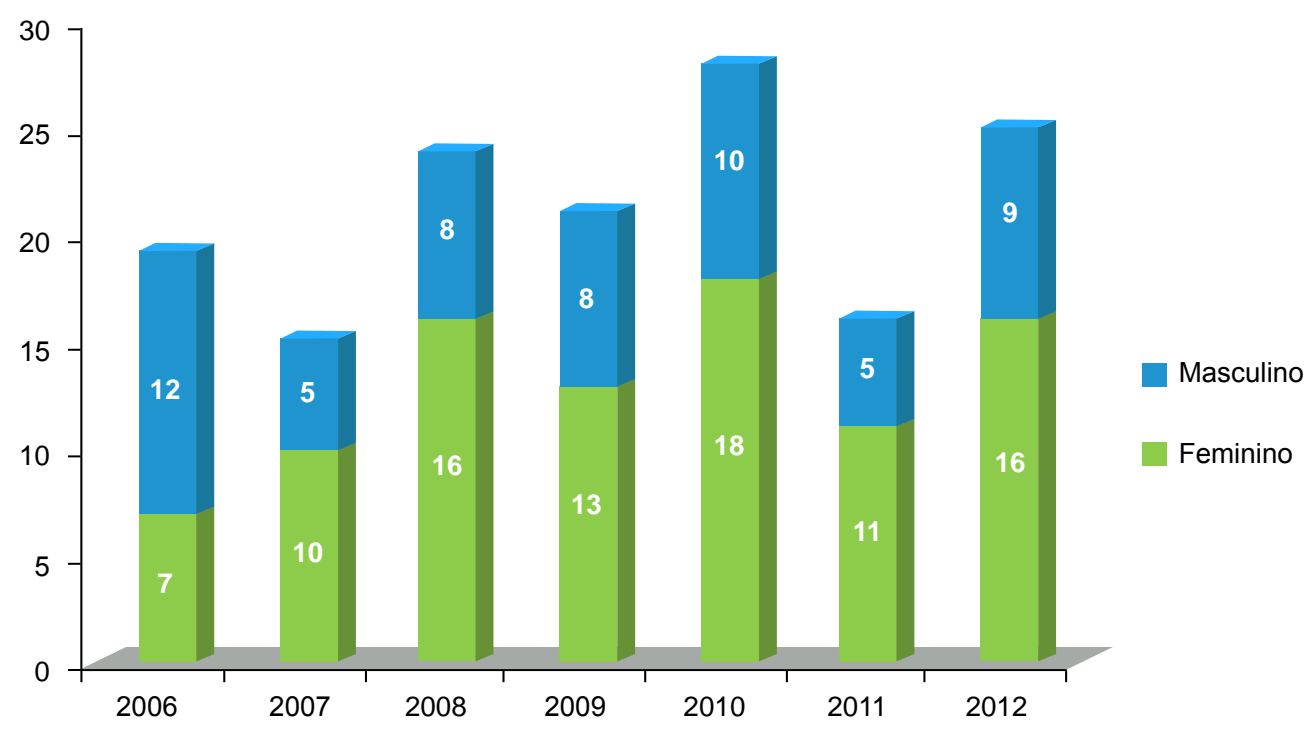

Figura 1 - Número de casos de melanoma cutâneo primário diagnosticados no CHSJ, distribuídos pelo género 
Tabela 1 - Características clínico-patológicas dos doentes com melanoma cutâneo

\begin{tabular}{|c|c|c|c|}
\hline & $\begin{array}{c}\text { Masculino } \\
n(\%)\end{array}$ & $\begin{array}{c}\text { Feminino } \\
\qquad n(\%)\end{array}$ & $\begin{array}{l}\text { Total } \\
n(\%)\end{array}$ \\
\hline \multicolumn{4}{|l|}{ Faixa etária } \\
\hline$\leq 30$ & $3(5,3)$ & $3(3,3)$ & $6(4,0)$ \\
\hline $31-40$ & $5(8,8)$ & $9(9,9)$ & $14(9,5)$ \\
\hline $41-50$ & $9(15,8)$ & $16(17,6)$ & $25(16,9)$ \\
\hline $51-60$ & $11(19,3)$ & $17(18,7)$ & $28(18,9)$ \\
\hline $61-70$ & $13(22,8)$ & $12(13,2)$ & $25(16,9)$ \\
\hline $71-80$ & $15(26,3)$ & $20(22,0)$ & $35(23,6)$ \\
\hline$>80$ & $1(1,7)$ & $14(15,4)$ & $15(10,1)$ \\
\hline \multicolumn{4}{|l|}{ Localização } \\
\hline Cervico-cefálica & $8(14,0)$ & $17(18,7)$ & $25(16,9)$ \\
\hline Tronco & $28(49,1)$ & $21(23,1)$ & $49(33,1)$ \\
\hline Membros superiores & $6(10,5)$ & $13(14,3)$ & $19(12,8)$ \\
\hline Membros inferiores & $15(26,3)$ & $40(43,9)$ & $55(37,2)$ \\
\hline \multicolumn{4}{|l|}{ Subtipo Histológico } \\
\hline SSM & $29(50,9)$ & $39(42,8)$ & $68(45,9)$ \\
\hline NM & $14(24,6)$ & $16(17,6)$ & $30(20,3)$ \\
\hline ALM & $9(15,8)$ & $15(16,7)$ & $24(16,2)$ \\
\hline LMM & $4(7,0)$ & $19(21,1)$ & $23(15,5)$ \\
\hline Outros & $1(1,7)$ & $2(2,2)$ & $3(2,0)$ \\
\hline \multicolumn{4}{|l|}{ Espessura tumoral } \\
\hline In situ & $12(21,4)$ & $15(16,7)$ & $27(18,5)$ \\
\hline$\leq 1 \mathrm{~mm}$ & $15(26,8)$ & $36(40,0)$ & $51(34,9)$ \\
\hline $1,01-2,0$ & $7(12,5)$ & $11(12,2)$ & $18(12,3)$ \\
\hline $2,01-4,0$ & $8(14,3)$ & $19(21,1)$ & $27(18,5)$ \\
\hline$>4,0$ & $14(25,0)$ & $9(10,0)$ & $23(15,8)$ \\
\hline Não avaliado & 1 & 1 & 2 \\
\hline \multicolumn{4}{|l|}{ Nível Clark } \\
\hline 1 & $12(21,8)$ & $15(17,2)$ & $27(19,0)$ \\
\hline II & $11(20,0)$ & $31(35,6)$ & $42(29,6)$ \\
\hline III & $10(18,2)$ & $21(24,1)$ & $31(21,8)$ \\
\hline IV & $15(27,3)$ & $13(15,0)$ & $28(19,7)$ \\
\hline $\mathrm{V}$ & $7(12,7)$ & $7(8,0)$ & $14(9,8)$ \\
\hline Não avaliado & 2 & 4 & 6 \\
\hline \multicolumn{4}{|c|}{ Índice Mitótico ( $\mathrm{n}^{\circ}$ mitoses $/ \mathrm{mm}^{2}$ ) } \\
\hline$<1$ & $3(6,7)$ & $8(10,8)$ & $11(9,2)$ \\
\hline $1-6$ & $29(64,4)$ & $52(70,3)$ & $81(68,1)$ \\
\hline$>6$ & $13(28,9)$ & $14(18,9)$ & $27(22,7)$ \\
\hline Não avaliado & 12 & 17 & 29 \\
\hline \multicolumn{4}{|l|}{ Ulceração } \\
\hline Ausente & $43(75,4)$ & $72(79,1)$ & $115(77,8)$ \\
\hline Presente & $14(24,6)$ & $19(20,9)$ & $33(22,3)$ \\
\hline \multicolumn{4}{|l|}{ Metastização } \\
\hline Sim & $12(26,1)$ & $8(10,4)$ & $20(16,3)$ \\
\hline Não & $34(73,9)$ & $69(89,6)$ & $103(83,7)$ \\
\hline Desconhecido & 11 & 14 & 25 \\
\hline \multicolumn{4}{|l|}{ Estádio } \\
\hline 0 & $12(21,4)$ & $15(16,7)$ & $27(18,5)$ \\
\hline IA & $11(19,6)$ & $24(26,7)$ & $35(24,0)$ \\
\hline IB & $9(16,1)$ & $18(20,0)$ & $27(18,5)$ \\
\hline IIA & $5(8,9)$ & $8(8,9)$ & $13(8,9)$ \\
\hline IIB & $2(3,6)$ & $7(7,8)$ & $9(6,2)$ \\
\hline IIC & $2(3,6)$ & $3(3,3)$ & $5(3,4)$ \\
\hline IIIA & $1(1,8)$ & $2(2,2)$ & $3(2,1)$ \\
\hline IIIB & $1(1,8)$ & $5(5,6)$ & $6(4,1)$ \\
\hline IIIC & $3(5,4)$ & $2(2,2)$ & $5(3,4)$ \\
\hline IV & $10(17,9)$ & $6(6,7)$ & $16(11,0)$ \\
\hline Não avaliado & 1 & 1 & 2 \\
\hline
\end{tabular}




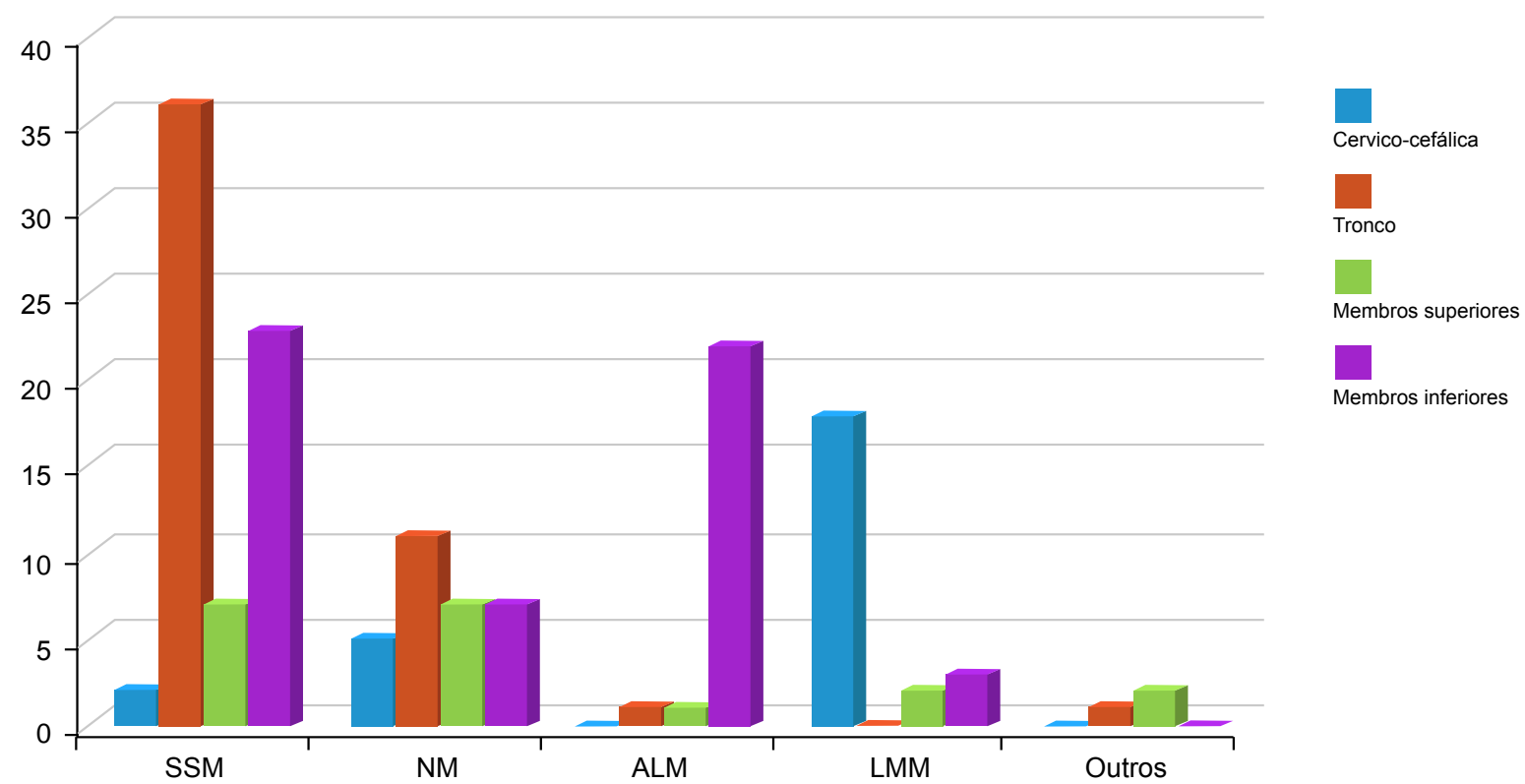

Figura 2 - Distribuição do melanoma maligno cutâneo de acordo com o subtipo histológico e localização anatómica

Em relação à topografia das lesões, as localizações mais comuns foram os membros inferiores $(37,2 \%)$, seguidos pelo tronco $(33,1 \%)$, região cervico-cefálica $(16,9 \%)$ e membros superiores $(12,8 \%)$ (Tabela 1). No sexo masculino, os melanomas ocorreram, preferencialmente, no dorso $(35,1 \%)$ e no feminino, predominaram nas pernas $(20,9 \%)$. Em $66,9 \%$ dos casos foi o doente que notou a presença da lesão, em $23,6 \%$ dos casos esta foi detetada pelo médico de família, $8,1 \%$ foram referenciados ou eram seguidos em Dermatologia por outro motivo e $1,4 \%$ dos doentes foram enviados por médico hospitalar de outra Especialidade.

O tempo médio de evolução da lesão foi de $1 \pm 1$ ano (2 meses a 5 anos). Em 19,6\% dos casos não há registo do tempo de evolução. As alterações mais frequentemente reportadas que motivaram a consulta foram, por ordem decrescente: aumento de dimensões de lesão pré-existente ( $41 \%)$, seguida de aparecimento de lesão de novo suspeita (24\%), alteração da cor (17\%), ulceração/hemorragia (9\%) e outros, nomeadamente, dor e prurido em $9 \%$.

Neste estudo, $18 \%$ dos doentes tinham nevos melanocíticos atípicos: $12 \%$ em quantidade inferior ou igual a 10 e, $6 \%$ apresentavam mais de 10 nevos atípicos. Em 48 processos clínicos não foi possível coletar esta informação.

Relativamente a história de cancro cutâneo não melanoma, esta constava em $8,8 \%$ dos doentes (nove mulheres e quatro homens), sendo a maioria relativa a carcinomas basocelulares $(77 \%)$ e os restantes a espinocelulares. A média de idades destes doentes foi de $72 \pm 7,7$ anos. Apresentavam queratoses actínicas 16 doentes, correspondentes a 12 mulheres e quatro homens, com a média etária de $72 \pm 9,8$ anos.

Apenas um doente tinha antecedentes pessoais de melanoma, ocorrido sete anos antes, e um outro mencionou história familiar num parente de $1^{\circ} \mathrm{grau}$. No que concerne a imunossupressão, dois doentes eram transplantados re- nais e um era portador de infecção VIH/SIDA.

O subtipo histológico de extensão superficial predominou em ambos os sexos $(45,9 \%)$ e teve como localização preferencial o tronco $(52,9 \%)$. O melanoma nodular foi identificado em $20,3 \%$ dos doentes e foi, igualmente, mais observado no tronco (36,7\%). O melanoma acrolentiginoso representou $16,2 \%$ dos casos diagnosticados e predominou nas extremidades inferiores (Fig. 2). O lentigo maligno melanoma registou-se em $15,5 \%$ dos casos, sendo a face o local mais envolvido $(65,2 \%)$. Outros tipos de melanoma foram o desmoplásico $(n=1)$, o mixoide $(n=1)$ e o spitzoide $(n=1)$.

O melanoma de extensão superficial predominou em todas as faixas etárias, com a excepção dos doentes com mais de 80 anos, em que se verificou um predomínio do tipo nodular (Fig. 3).

Relativamente à espessura tumoral, houve um predomínio dos melanomas considerados finos, com índice de Breslow $\leq 1 \mathrm{~mm}(53,4 \%)$, sendo que $18,5 \%$ da totalidade dos casos eram in situ (Tabela 1). A média do índice de Breslow foi de 2,6 $\pm 3,2 \mathrm{~mm}(0,1-22 \mathrm{~mm})$ e a maior espessura foi observada no melanoma do tipo nodular (índice de Breslow médio: $5,4 \pm 4,5 \mathrm{~mm}$ ).

Dos 148 casos, 142 tinham informação quanto ao nível de invasão tumoral de Clark, tendo a maioria sido classificada no nível II de Clark (29,6\%) (Tabela 1$)$.

$O$ índice mitótico ( $\mathrm{n}^{\circ}$ de mitoses/ $\mathrm{mm}^{2}$ ) foi calculado em 119 doentes (de notar que não foram avaliados 29 casos, incluindo os 27 casos de melanoma in situ), tendo-se verificado um predomínio entre 1 - 6 mitoses/ $\mathrm{mm}^{2}(68,1 \%$ ) (Tabela 1).

A ulceração esteve presente em 33 doentes (19 muIheres e 14 homens) e predominou nos melanomas com índice de Breslow superior a $2 \mathrm{~mm}(87,9 \%)$ e nos subtipos nodular $(54,5 \%)$ e acrolentiginoso $(30,3 \%)$. Não se obser- 


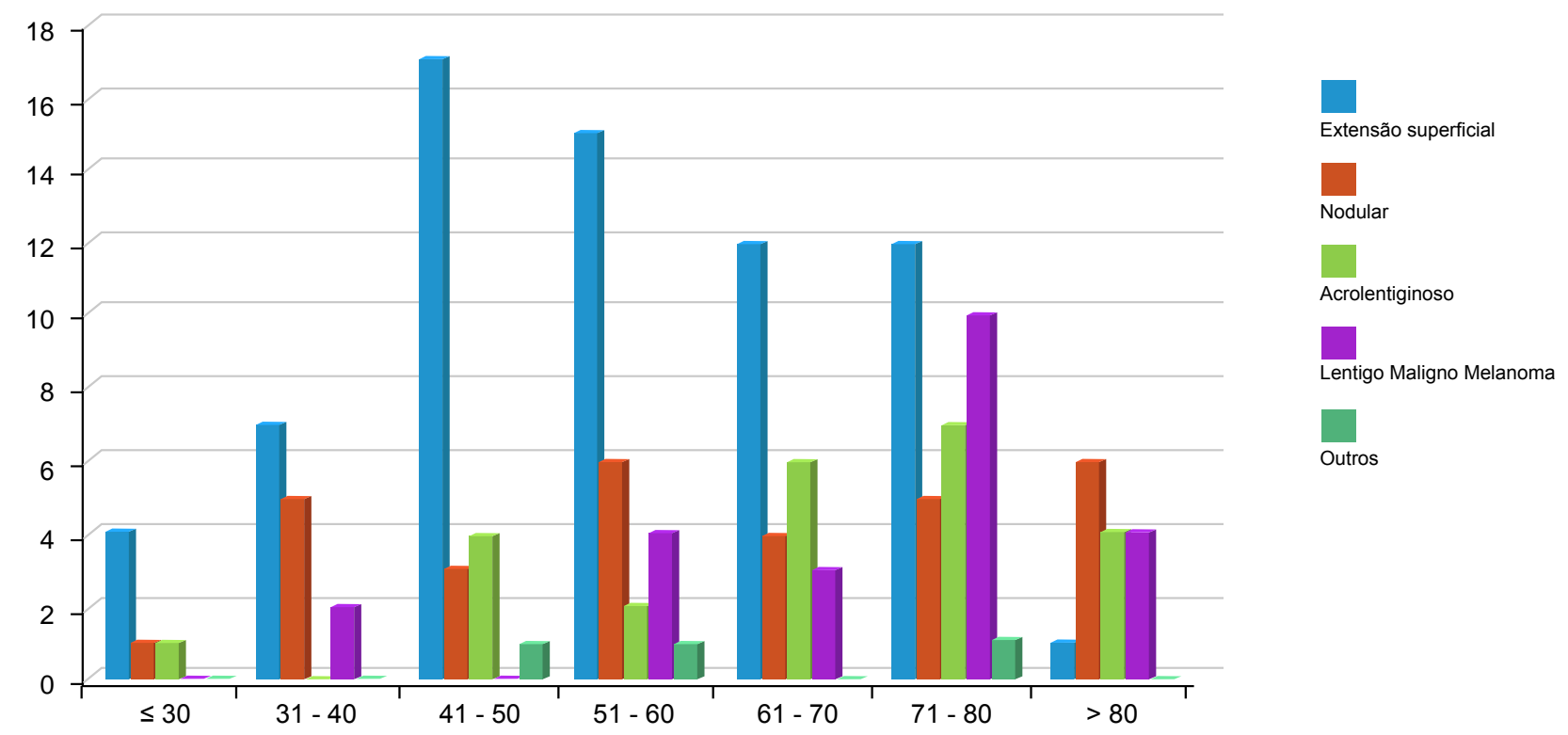

Figura 3 - Distribuição do melanoma maligno cutâneo de acordo com o subtipo histológico e grupo etário

vou diferença estatisticamente significativa entre ulceração e as variáveis sexo, idade e localização anatómica.

Quanto a outros parâmetros histológicos, observou-se a presença de infiltrado inflamatório linfocitário em 17,6\% dos casos, sinais de regressão em $7,4 \%$, nódulos satélites em $1,4 \%$, imagens de invasão vascular linfática em $7,4 \%$ e perineural em $0,7 \%$.

Analisando a relação entre os diferentes grupos de espessura tumoral e a localização anatómica do melanoma verifica-se que os melanomas finos predominaram no tronco $(37,2 \%, 29 / 78)$ enquanto os tumores com espessura superior a $1 \mathrm{~mm}$ predominaram nos membros inferiores $(50 \%$, 34/68) (Tabela 2).

Os tumores espessos (índice de Breslow $>4 \mathrm{~mm}$ ) foram predominantemente do tipo nodular $(69,5 \%, 16 / 23)$.

No que concerne ao tratamento, todos os doentes foram submetidos a alargamento das margens cirúrgicas, com a excepção de quatro doentes que recusaram cirurgia.

A pesquisa do gânglio sentinela foi efetuada em 63 doentes, dos quais 50 apresentavam espessura intermédia (índice de Breslow entre 1 e $4 \mathrm{~mm}$ ), 11 tinham índice de Breslow superior a $4 \mathrm{~mm}$ e em dois doentes o índice de Breslow foi inferior a $1 \mathrm{~mm}$. Na globalidade, o índice de Breslow médio foi de $2,8 \pm 1,9 \mathrm{~mm}$. A pesquisa de gânglio sentinela foi positiva em 16 doentes $(25,4 \%)$, tendo motivado o esvaziamento ganglionar.

Os melanomas foram estadiados de acordo com as guidelines AJCC, 2010, tendo-se verificado que o maior $\mathrm{n}^{\circ}$ de doentes se encontrava no estádio IA (24,0\%) (Tabela 1).

O período médio de seguimento foi de $35 \pm 22$ meses (5 a 90 meses). Durante esse período, $78,8 \%$ dos doentes mantiveram-se livres de doença, $4,9 \%$ sofreram recidiva local e 16,3\% desenvolveram metástases. Em 25 casos não foi possível avaliar a evolução, por abandono da consulta. A recidiva local e a metastização ocorreram, em média, respetivamente, $14,7 \pm 7,2$ e 19,3 $\pm 17,8$ meses após o diagnóstico. Em relação às metástases, os locais mais envolvidos foram, por ordem decrescente: gânglios linfáticos $(26 \%)$, pulmão $(24 \%)$, tegumento cutâneo $(19 \%)$, cérebro $(9 \%)$ e fígado $(7 \%)$.

O índice de Breslow médio para o grupo de doentes em que ocorreu metastização foi de $5,4 \pm 4,8 \mathrm{~mm}$, enquanto para o grupo que não desenvolveu metástases, foi de 1,82 $\pm 1,9 \mathrm{~mm}$ ( $p=0,003$, teste Welch). Os tumores espessos (índice de Breslow $>4 \mathrm{~mm}$ ) tiveram maior risco de evoluir para metastização $(p<0,001)$.

A associação entre ulceração e metastização foi estatisticamente significativa $(p<0,001)$, sendo que os doentes com ulceração tiveram um risco acrescido 19 vezes maior de desenvolver metástases (IC 95\%: 6,0 - 60,7). Efetuando a análise multivariada, a ulceração manteve-se como fator de risco independente para metastização OR 5,5 (IC 95\%: $1,5-20,8)$.

De igual modo verificou-se uma associação estatisticamente significativa entre a positividade do gânglio sentinela e a metastização $(p=0,002)$. De facto, os doentes com gânglio sentinela positivo tiveram um risco acrescido de 8,7 vezes maior de evoluírem com metastização, comparativamente aos doentes em que a sua pesquisa foi negativa (IC $95 \%: 2,2-34,4)$.

Não se observou diferença estatisticamente significativa entre a localização anatómica do melanoma e a ocorrência de metástases.

Os doentes que evoluíram com metástases foram orientados para a consulta de Oncologia Médica, tendo as opções terapêuticas sido ajustadas a cada caso, e incluíram o esvaziamento ganglionar, tratamento adjuvante com IFNa $2 b$, quimioterapia (dacarbazina, temozolamida e fotemustina), radioterapia e perfusão hipertérmica do membro (realizada no IPO, Porto). 
Tabela 2 - Características clínico-patológicas do melanoma de acordo com a espessura tumoral

\begin{tabular}{|c|c|c|c|c|c|c|}
\hline \multirow[b]{2}{*}{ Variável } & \multicolumn{5}{|c|}{ Espessura tumoral } & \multirow[b]{2}{*}{ Valor $p$} \\
\hline & $\begin{array}{l}\text { In situ } \\
\text { n (\%) }\end{array}$ & $\begin{array}{c}\leq 1 \mathrm{~mm} \\
n(\%)\end{array}$ & $\begin{array}{c}1,01-2,0 \mathrm{~mm} \\
n(\%)\end{array}$ & $\begin{array}{c}2,1 \text { - } 4,0 \mathrm{~mm} \\
n(\%)\end{array}$ & $\begin{array}{c}>4 \mathrm{~mm} \\
n(\%)\end{array}$ & \\
\hline \multicolumn{7}{|l|}{ Sexo } \\
\hline Masculino & $12(44,4 \%)$ & $15(29,4 \%)$ & $7(38,9 \%)$ & $8(29,6 \%)$ & $14(60,9 \%)$ & \multirow[t]{2}{*}{0,074} \\
\hline Feminino & $15(55,6 \%)$ & $36(70,6 \%)$ & $11(61,1 \%)$ & $19(70,4 \%)$ & $9(39,1 \%)$ & \\
\hline \multicolumn{7}{|l|}{ Faixa etária } \\
\hline$\leq 30$ & $1(3,7 \%)$ & $3(5,9 \%)$ & $1(5,6 \%)$ & 0 & $1(4,4 \%)$ & \multirow{7}{*}{ * } \\
\hline $31-40$ & $5(18,5 \%)$ & $4(7,8 \%)$ & $1(5,6 \%)$ & $1(3,7 \%)$ & $3(13,0 \%)$ & \\
\hline $41-50$ & $7(25,9 \%)$ & $9(17,6 \%)$ & $3(16,7 \%)$ & $3(11,1 \%)$ & $2(8,7 \%)$ & \\
\hline $51-60$ & $4(14,8 \%)$ & $12(23,5 \%)$ & $4(22,2 \%)$ & $4(14,8 \%)$ & $4(17,4 \%)$ & \\
\hline $61-70$ & $3(11,1 \%)$ & $10(19,6 \%)$ & $5(27,7 \%)$ & $4(14,8 \%)$ & $3(13,0 \%)$ & \\
\hline $71-80$ & $4(14,8 \%)$ & $10(19,6 \%)$ & $4(22,2 \%)$ & $9(33,3 \%)$ & $7(30,4 \%)$ & \\
\hline$>80$ & $3(11,1 \%)$ & $3(5,9 \%)$ & 0 & $6(22,2 \%)$ & $3(13,0 \%)$ & \\
\hline \multicolumn{7}{|l|}{ Localização } \\
\hline Cervico/cefálica & $8(29,6 \%)$ & $10(19,6 \%)$ & 0 & $2(7,4 \%)$ & $5(21,7 \%)$ & \multirow{4}{*}{ * } \\
\hline Tronco & $7(25,9 \%)$ & $22(43,1 \%)$ & $7(38,9 \%)$ & $5(18,5 \%)$ & $7(30,4 \%)$ & \\
\hline MS & $3(11,1 \%)$ & $7(13,7 \%)$ & $1(5,6 \%)$ & $2(7,4 \%)$ & $5(21,7 \%)$ & \\
\hline $\mathrm{MI}$ & $9(33,3 \%)$ & $12(23,5 \%)$ & $10(55,5 \%)$ & $18(66,7 \%)$ & $6(26,1 \%)$ & \\
\hline \multicolumn{7}{|l|}{ Tipo histológico } \\
\hline SSM & $11(40,7 \%)$ & $32(62,7 \%)$ & $12(66,7 \%)$ & $8(29,6 \%)$ & $4(17,4 \%)$ & \multirow{5}{*}{ * } \\
\hline NM & 0 & $2(3,9 \%)$ & $5(27,7 \%)$ & $7(25,9 \%)$ & $16(69,5 \%)$ & \\
\hline ALM & $5(18,5 \%)$ & $5(9,8 \%)$ & $1(5,6 \%)$ & $11(40,7 \%)$ & $2(8,7 \%)$ & \\
\hline LMM & $11(40,7 \%)$ & $11(21,6 \%)$ & 0 & $1(3,7 \%)$ & 0 & \\
\hline Outros & 0 & $1(2 \%)$ & 0 & 0 & $1(4,4 \%)$ & \\
\hline \multicolumn{7}{|l|}{ Ulceração } \\
\hline Presente & 0 & $1(2 \%)$ & $3(16,7 \%)$ & $14(51,9 \%)$ & $15(65,2 \%)$ & \multirow{2}{*}{$<0,001$} \\
\hline Ausente & $27(100 \%)$ & $50(98,0 \%)$ & $15(83,3 \%)$ & $13(48,1 \%)$ & $8(34,8 \%)$ & \\
\hline \multicolumn{7}{|l|}{ Metástases } \\
\hline Sim & 0 & 1 & 1 & 7 & 11 (57,9\%) & \multirow{2}{*}{$<0,001$} \\
\hline Não & 21 & 40 & 16 & 16 & 8 & \\
\hline
\end{tabular}

* não foi possível calcular $p$

SSM- melanoma de extensão superficial; NM- melanoma nodular; ALM- melanoma acrolentiginoso; LMM- lentigo maligno melanoma.

Quinze doentes (5 mulheres e 10 homens) faleceram em consequência do melanoma. A média etária desses doentes foi de 66,5 $\pm 12,6$ anos (44-88 anos) e o índice de Breslow médio foi de $6,1 \pm 5,3 \mathrm{~mm}(1,8-22 \mathrm{~mm})$. O óbito ocorreu, em média 23,6 \pm 17,2 meses após o diagnóstico (10-54 meses). Não houve associação estatisticamente significativa entre sexo e óbito.

\section{DISCUSSÃO}

A incidência do melanoma tem vindo a aumentar em vários países. A maior incidência verifica-se no continen- te australiano, seguido pelo continente norte-americano. ${ }^{7}$ Também na Europa se tem verificado este aumento, nomeadamente, na Comunidade Europeia, o melanoma já representa 1 e $1,8 \%$ de todos os tumores malignos, respetivamente, no sexo masculino e feminino. ${ }^{8}$

Em Portugal, e de acordo com dados do registo oncológico nacional (RON, 2006), a incidência do melanoma é de 722 novos casos/ano, correspondente a 6,8 casos/ 100 000 habitantes. Esta incidência não difere muito daquela encontrada nos restantes países do Sul da Europa, sendo bastante similar à de Espanha, ligeiramente superior à da 
Grécia e inferior à de Itália ${ }^{9}$

Neste estudo, o número de casos de melanoma oscilou ao longo destes sete anos, com uma média de 21 casos/ ano.

A principal razão apontada para o aumento da incidência do melanoma é a modificação dos estilos de vida, traduzidos num acréscimo de exposição à radiação ultravioleta. ${ }^{1-11} \mathrm{O}$ papel da redução da camada de ozono é menos claro. No entanto, alguns estudos sugerem que uma redução em $1 \%$ da camada de ozono leva ao aumento da incidência do melanoma, na ordem dos $0,6 \% .^{12}$ Por outro lado, uma maior acuidade diagnóstica, bem como o aumento da esperança de vida poderão contribuir para o crescimento do número de casos diagnosticados. ${ }^{13,14}$

Neste estudo, e em concordância com o que se verifica a nível nacional $\left.\right|^{9,15,16}$ houve um predomínio de mulheres, com uma relação entre sexos de 1,6:1. Esta predominância foi observada em quase todas as faixas etárias.

A média de idades na altura do diagnóstico foi de 61 anos, com a maioria dos doentes pertencendo ao grupo etário entre os 71 e os 80 anos (23,6\%). Apenas se encontrou um caso de melanoma em doente com idade inferior a 18 anos, confirmando a raridade da sua ocorrência em idade pediátrica. ${ }^{17}$

De acordo com a literatura, a média etária é de 55 anos de idade, ${ }^{7}$ mas há uma incidência crescente entre os idosos, resultado do aumento da esperança de vida, pelo que se prevê que esta média etária venha a aumentar. ${ }^{18}$

As localizações mais frequentemente registadas foram os membros inferiores $(37,1 \%)$ e o tronco $(33,1 \%)$. No sexo masculino, o dorso foi o local mais afetado $(35,1 \%)$, enquanto no sexo feminino as lesões ocorreram preferencialmente nas pernas (20,9\%). Estes dados estão em consonância com o descrito na literatura. ${ }^{5,14,16,19} \mathrm{~A}$ predominância de melanomas no dorso dos homens e nas pernas das mulheres, áreas de pele intermitentemente expostas ao sol, parece demonstrar quão importante é a contribuição do fator comportamental no surgimento do melanoma. ${ }^{20}$

À semelhança da literatura, predominou o subtipo histológico de extensão superficial $(45,9 \%)$, seguido pelo melanoma nodular $(20,3 \%)$, ambos mais comuns no tronco. Contrariamente ao observado noutras séries nacionais ${ }^{15,16}$ e europeias, ${ }^{21,22}$ o melanoma do tipo acrolentiginoso foi neste estudo, o terceiro tipo de melanoma mais comum com uma frequência de $16,2 \%$, ultrapassando o lentigo maligno melanoma (15,5\%). Ele predominou nos idosos, tendo a média de idades sido de $65 \pm 17,3$ anos e o índice de Breslow médio de 2,5 $\pm 1,4 \mathrm{~mm}$. Apesar de ser pouco frequente nos caucasianos (2-8\%), é no entanto, o tipo de melanoma com maior prevalência nos negros (até 70\%) e asiáticos (até $45 \%$ ). $., 23,24$ Neste estudo todos os doentes eram caucasianos, mas não foi possível obter informação relativa ao fototipo.

O risco de melanoma aumenta na presença de nevos melanocíticos atípicos. De facto, Tucker et $a^{25}$ concluíram que a presença de um único nevo atípico duplica o risco de melanoma, enquanto 10 ou mais nevos atípicos associam- -se a um risco 12 vezes superior. Nesta série, $18 \%$ dos doentes apresentavam nevos melanocíticos atípicos, valor semelhante ao descrito na literatura. ${ }^{26}$

É comum a associação entre melanoma maligno e cancro cutâneo não melanoma, incluindo os carcinomas basocelular e espinocelular, uma vez que as três neoplasias se relacionam com a exposição solar. ${ }^{27}$ Nesta casuística, 8,8\% dos doentes tinham história de um outro cancro cutâneo não melanoma e 10,8\% apresentavam queratoses actínicas.

A espessura tumoral (índice de Breslow) constitui o fator de prognóstico mais relevante, sendo importante para avaliar a sobrevida, o risco de recidiva local ou de metastização, e é fundamental na abordagem terapêutica, uma vez que determina as margens de exérese cirúrgica, como também a indicação para pesquisa do gânglio sentinela. ${ }^{28-31}$

No presente estudo, houve predomínio dos melanomas finos (índice de Breslow $\leq 1 \mathrm{~mm}$ ), considerados de prognóstico mais favorável. As mulheres, bem como os doentes com idade inferior a 65 anos, apresentaram uma maior proporção de melanomas finos (respetivamente $56,7 \%$ e $60,7 \%$ ), enquanto nos homens e nos doentes com idade igual ou superior a 65 anos observou-se uma maior proporção de melanomas com espessura superior a $1 \mathrm{~mm}$ (respetivamente $51,8 \%$ e $55,2 \%$ ), o que está em concordância com a literatura. ${ }^{17,32,33}$

É possível, que esta diferença se deva ao facto de as mulheres prestarem maior atenção às alterações da sua pele e procurarem cuidados médicos dermatológicos mais cedo e mais frequentemente, para além de parecer aderirem com maior regularidade às campanhas de rastreio, resultando num diagnóstico mais precoce.$^{17}$ Pelo contrário, os idosos têm maior dificuldade em reconhecer ou detetar alterações na sua pele, pelo que atrasam a procura de cuidados médicos. ${ }^{34}$

Os melanomas dos tipos de extensão superficial e lentigo maligno melanoma eram predominantemente finos (respetivamente $64,1 \%$ e $95,6 \%$ ), enquanto a maioria dos melanomas dos tipos nodular $(93,3 \%)$ e acrolentiginoso $(58,3 \%)$ tinham um índice de Breslow superior a $1 \mathrm{~mm}(p$ $<0,001)$. Esta observação era expectável, pois os tipos de extensão superficial e o lentigo maligno melanoma possuem uma fase de crescimento radial mais prolongada, comparativamente a outros subtipos histológicos. ${ }^{17}$

Estudos epidemiológicos de vários países demonstram uma incidência crescente dos melanomas finos e uma estabilização dos melanomas espessos..$^{31-35} \mathrm{~A}$ explicação plausível é a deteção mais precoce do melanoma, em estádios iniciais, o que também justifica que o aumento da incidência seja proporcionalmente maior do que a mortalidade, com uma clara melhoria na sobrevida. ${ }^{4,36}$ De igual modo, publicações recentes reportam um predomínio de níveis baixos de Clark (I, II e III) ${ }^{37}$ em paralelo com o encontrado no presente trabalho, refletindo, mais uma vez, a maior atenção para o diagnóstico precoce do melanoma nos últimos anos.

A seguir ao índice de Breslow, o índice mitótico é o 
segundo mais importante preditor independente de sobrevivência, em doentes com melanoma. ${ }^{38,39}$ A presença de mitoses na derme está associada a uma diminuição da sobrevida. Quando o número de mitoses é igual ou superior a seis, o risco de metastização é 12 vezes superior ao de um doente em cujo tumor não se observam mitoses. ${ }^{40}$ Nesta casuística, todos os melanomas que metastizaram, tinham um índice mitótico superior a 1 mitose/ $\mathrm{mm}^{2}(p=0,013)$.

A ulceração é um outro parâmetro clínico-histológico associado a uma maior agressividade tumoral. ${ }^{41}$ Entre os melanomas finos, a ulceração ocorreu em apenas $1 \%$ desses tumores, enquanto $53 \%$ dos melanomas com espessura superior a $1 \mathrm{~mm}$ manifestavam esta alteração.

Depois de confirmado o diagnóstico, foi efetuado o alargamento das margens cirúrgicas, e neste hospital, regra geral, naqueles doentes com tumor de espessura intermédia, entre 1 e $4 \mathrm{~mm}$, realiza-se a pesquisa e biópsia do gânglio sentinela. Esta pesquisa pode ainda ser considerada no melanoma com índice de Breslow entre 0,76 e 0,99 mm, na presença de ulceração, idade jovem, índice mitótico $\geq 1 /$ $\mathrm{mm}^{2} \mathrm{e}$ invasão angiolinfática. ${ }^{5} \mathrm{~A}$ biópsia do gânglio sentinela providencia informação mais segura para o estadiamento e é relevante no planeamento terapêutico, nomeadamente, identifica os candidatos a linfadenectomia terapêutica e selecciona os doentes com indicação para terapêutica adjuvante. ${ }^{42,43}$ A pesquisa do gânglio sentinela, quando positiva e seguida de linfadenectomia terapêutica, demonstrou benefícios na sobrevida daqueles doentes comparativamente aos submetidos a linfadenectomia somente após evidência clínica de doença ganglionar. ${ }^{42}$

Dos 123 doentes que se mantiveram em seguimento, 20 doentes $(16,3 \%)$ evoluíram com metástases, as quais ocorreram, em média, 19 meses após o diagnóstico. Nos

\section{REFERÊNCIAS}

1. Garbe C, Bauer J. Melanoma. In: Bolognia JL, Jorizzo JL, Schaffer JV, editors. Dermatology. $3^{a}$ ed. New York: Elsevier; 2012. p.1885-914.

2. Jemal A, Siegel R, Ward E, Hao Y, Xu J, Thun MJ. Cancer statistics, 2009. CA Cancer J Clin. 2009;59:225-49.

3. Parkin DM, Bray F, Ferlay J, Pisani P. Estimating the world cancer burden: Globocan 2000. Int J Cancer. 2001;94:153-6.

4. Mackie RM, Hauschild A, Eggermont AM. Epidemiology of invasive cutaneous melanoma. Ann Oncol. 2009:20:vi1-7.

5. Bailey E, Sober A, Tsao H, Mihm M, Johnson T. Cutaneous melanoma. In: Goldsmith L, Katz S, Gilchrest B, Paller A, Leffell D, Wolff K editors. Fitzpatrick's Dermatology in General Medicine. $8^{\text {th }}$ ed. Philadelphia: McGraw-Hill; 2012. p.1416-44.

6. Houghton NA, Polsky D. Focus on melanoma. Cancer Cell. 2002;2:27578.

7. Garbe C, Leiter U. Melanoma epidemiology and trends. Clin Dermatol. 2009;27:3-9.

8. De Braud F, Khayat D, Kroon BB, Valdagni R, Bruzzi P, Cascinelli N. Malignant melanoma. Crit Rev Oncol Hematol. 2003;47:35-63.

9. Forsea AM, Del Marmol V, de Vries E, Bailey E, Geller AC. Melanoma incidence and mortality in Europe. Br J Dermatol. 2012;167:1124-30.

10. Katsambas A, Nicolaidou E. Cutaneous malignant melanoma and sun exposure. Recent developments in epidemiology. Arch Dermatol. 1996;132:444-50.

11. Brochez L, Naeyaert JM. Understanding the trends in melanoma incidence and mortality: where do we stand? Eur J Dermatol. 2000;10:70-5.

12. Burton RC, Armstrong BK. Recent incidence trends imply nonmetastasizing form of invasive melanoma. Melanoma Res. 1994;4:107-13.

13. Erdei E, Torres S. A new understanding in epidemiology of melanoma. Expert Rev Anticancer Ther. 2010;10:1811-23. melanomas finos predominou a ausência de metástases, o que está de acordo com a literatura, considerando-se tumores de melhor prognóstico. ${ }^{44}$

\section{CONCLUSÃo}

Apesar de estarem disponíveis novas opções terapêuticas e outras em vias de desenvolvimento, especialmente para o melanoma avançado, o diagnóstico precoce do melanoma maligno permanece a pedra basilar mais eficaz para aumentar a sobrevida dos doentes. A identificação dos grupos de maior risco e a compreensão do comportamento histológico do melanoma, bem como dos seus fatores de prognóstico, são igualmente importantes para delinear a melhor estratégia de abordagem populacional.

Em Portugal já foram realizados alguns estudos de caracterização epidemiológica do melanoma, no entanto, são ainda escassos, e a informação fornecida pelos registos oncológicos nacionais é limitada.

O predomínio de melanomas finos verificado no presente estudo parece indicar uma possível precocidade diagnóstica, no entanto, e de acordo com as estimativas dos registos oncológicos, ${ }^{45}$ a incidência do melanoma no nosso país prevê-se crescente, sendo fundamental apostar na prevenção.

\section{CONFLITOS DE INTERESSE}

Não existem, por parte dos Autores, conflitos de interesse a declarar.

\section{FONTES DE FINANCIAMENTO}

Não existem, por parte dos Autores, fontes de financiamento a declarar.

14. Lasithiotakis K, Leiter U, Meier F, Eigentler T, Metzler G, Moehrle M, et al. Age and gender are significant independent predictors of survival in primary cutaneous melanoma. Cancer. 2008;112:1795-804.

15. Ferreira M, Costa V, Torres $T$, Selores M. Análise retrospectiva de melanoma cutâneo primário: 1996-2006. Trab Soc Port Dermatol Venereol. 2007;65:509-18

16. Catorze MG, Cabeças MA, Rafael M, Chaveiro A, Lamarão P, Cardoso $\mathrm{J}$, et al. Malignant melanoma (epidemiological aspects of the casuistics in the department of dermatology of a Lisbon hospital - 1991-1997). Skin Cancer. 1998;13:75-80.

17. Oumeish OY. Epidemiology of primary cutaneous malignant melanoma in Jordan. Int J Dermatol. 1997;36:113-5.

18. Ferrari Júnior NM, Muller H, Ribeiro M, Maia M, Saches Júnior JA. Cutaneous melanoma: descriptive epidemiological study. São Paulo Med J 2008;126:41-7.

19. Ríos L, Nagore E, López JL, Redondo P, Martí RM, Fernández-de-Misa $\mathrm{R}$, et al. Melanoma characteristics at diagnosis from the Spanish $\mathrm{Na}-$ tional Cutaneous Melanoma Registry: 15 years of experience. Actas Dermosifiliogr. 2013;104:789-99.

20. Bakos L, Wagner M, Bakos RM, Leite CS, Sperhacke CL, Dzekaniak $\mathrm{KS}$, et al. Sunburn, sunscreens, and phenotypes: some risk factors for cutaneous melanoma in southern Brazil. Int J Dermatol. 2002;41:55762.

21. Nagore E, Oliver V, Botella-Estrada R, Moreno-Picot S, Guillén C Fortea JM. Clinicopathological analysis of 1571 cutaneous malignant melanomas in Valencia, Spain: factors related to tumour thickness. Acta Derm Venereol. 2006;86:50-6.

22. Chiarugi A, Nardini P, Crocetti E, Carli P, De Giorgi V, Borgognoni L, et al. Familial and sporadic melanoma: different clinical and histopathologi- 
cal features in the Italian population- a multicentre epidemiological study - by GIPMe (Italian Multidisciplinary Group on Melanoma). J Eur Acad Dermatol Venereol. 2012;26:194-9.

23. Cress RD, Holly EA: Incidence of cutaneous melanoma among nonhispanic whites, hispanics, asians, and blacks: an analysis of California Cancer Registry data, 1988-93. Cancer Causes Control. 1997;8:246-78.

24. Ishihara K, Saida T, Yamamoto A, Japanese Skin Cancer Society Prognosis and Statistical Investigations Committee. Updated statistical data for malignant melanoma in Japan. Int J Clin Oncol. 2001;6:109-16.

25. Tucker MA, Halpern A, Holly EA, Hartge P, Elder DE, Sagebiel RW, et al. Clinically recognized dysplastic nevi. A central risk factor for cutaneous melanoma. JAMA. 1997;277:1020-9.

26. Ferrone CR, Ben Porat L, Panageas KS, Berwick M, Halpern AC, Potel A, et al. Clinicopathological features and risk factors for multiple primary melanomas. JAMA. 2005;294:1647-54.

27. Armstrong BK, Kricker A. The epidemiology of UV induced skin cancers J Photochem Photobiol B. 2001;63:8-18.

28. Wagner JD, Gordon MS, Chuang TY, Coleman JJ. Current therapy of cutaneous melanoma. Plast Reconstr Surg. 2000;105:1774-99.

29. Breslow A. Thickness, cross-sectional areas and depth of invasion in the prognosis of cutaneous melanoma. Ann Surg. 1970;172:902-8.

30. Van Der Esch EP, Cascinelli N, Preda F, Morabito A, Bufalino R. Stage I melanoma of the skin: evaluation of prognosis according to histologic characteristics. Cancer. 1981;48:1668-73.

31. Balch CM, Murad TM, Soong SJ, Ingalls AL, Halpern NB, Maddox WA. A multifactorial analysis of melanoma: prognostic histopathological features comparing Clark's and Breslow staging methods. Ann Surg. 1978; $188: 732-42$

32. Jemal A, Devesa SS, Hartge P, Tucker MA. Recent trends in cutaneous melanoma incidence among whites in the United States. J Natl Cancer Inst. 2001;93:678-3.

33. Buettner PG, Leiter U, Eigentler TK, Garbe C. Development of prognostic factors and survival in cutaneous melanoma over 25 years. An analysis of the Central Malignant Melanoma Registry of the German Dermatological Society. Cancer. 2005;103:616-24.

34. Criscione VD, Weinstock MA. Melanoma thickness trends in the United States, 1988-2006. J Invest Dermatol. 2010;130:793-7.

35. Geller AC, Swetter SM, Brooks K, Demierre MF, Yaroch AL. Screening early detection, and trends for melanoma: current status (2000-2006) and future directions. J Am Acad Dermatol. 2007;57:555-72.

36. de Vries E, Coebergh JW. Cutaneous malignant melanoma in Europe. Eur J Cancer. 2004;40:2355-66.

37. Garbe C, McLeod GR, Buettner PG. Time trends of cutaneous melanoma in Queensland, Australia and Central Europe. Cancer. 2000;89:1269-78.

38. Azzola MF, Shaw HM, Thompson JF, Soong SJ, Scolyer RA, Watson GF, et al. Tumor mitotic rate is a more powerful prognostic indicator than ulceration in patients with primary cutaneous melanoma: an analysis of 3661 patients from a single center. Cancer. 2003;97:1488-98.

39. Barnhill RL, Katzen J, Spatz A, Fine J, Berwick M. The importance of mitotic rate as a prognostic factor for localized cutaneous melanoma. $\mathrm{J}$ Cutan Pathol. 2005;32:268-73.

40. Clark WH Jr, Elder DE, Guerry D 4th, Braitman LE, Trock BJ, Schultz $D$, et al. Model predicting survival in stage I melanoma based on tumor progression. J Natl Cancer Inst. 1989;81:1893-904.

41. Zettersten E, Shaikh L, Ramirez R, Kashani-Sabet M. Prognostic factors in primary cutaneous melanoma. Surg Clin North Am. 2003;83:61-75.

42. Morton DL, Thompson JF, Cochran AJ, Mozzillo N, Elashoff R, Essner $\mathrm{R}$, et al. Sentinel-node biopsy or nodal observation in melanoma. N Engl J Med. 2006;355:1307-17.

43. Rosenberg SA. Why perform sentinel-lymph-node biopsy in patients with melanoma? Nat Clin Pract Oncol. 2008;5:1.

44. Lang PG Jr. Malignant melanoma. Med Clin North Am. 1998;82:1325 58.

45. Instituto Português de Oncologia do Porto [homepage na Internet]; [consultado 2014 Abril 24]. Disponível em: http://www.ipoporto.min-saude. pt/. 


\section{Melanoma Maligno Cutâneo: Estudo Retrospetivo de Sete Anos (2006-2012) \\ Acta Med Port 2014:27:480-488}

Publicado pela Acta Médica Portuguesa, a Revista Científica da Ordem dos Médicos

Av. Almirante Gago Coutinho, 151

1749-084 Lisboa, Portugal.

Tel: +351 218428215

E-mail: submissao@actamedicaportuguesa.com

www.actamedicaportuguesa.com

ISSN:0870-399X | e-ISSN: 1646-0758

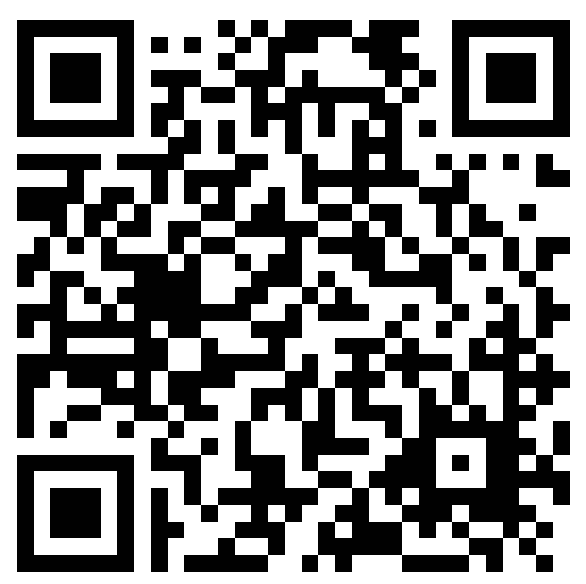

